\title{
Variability of phase and amplitude fronts due to horizontal refraction in shallow water
}

\author{
Boris G. Katsnelson ${ }^{\text {a) }}$ \\ Leon Charney School of Marine Sciences, University of Haifa, Mount Carmel, Haifa 3498838, Israel \\ Valery A. Grigorev \\ Voronezh State University, Universitetskaya sq 1, Voronezh 394018, Russia \\ James F. Lynch \\ Woods Hole Oceanographic Institution, 98 Water Street, MS No.12, Woods Hole, Massachusetts 02543, USA
}

(Received 16 July 2017; revised 8 December 2017; accepted 13 December 2017; published online 16 January 2018)

The variability of the interference pattern of a narrow-band sound signal in a shallow water waveguide in the horizontal plane in the presence of horizontal stratification, in particular due to linear internal waves, is studied. It is shown that lines of constant phase (a phase front) and lines of constant amplitude/envelope (an amplitude front) for each waveguide mode may have different directions in the spatial vicinity of the point of reception. The angle between them depends on the waveguide's parameters, the mode number, and the sound frequency. Theoretical estimates and data processing methodology for obtaining these angles from experimental data recorded by a horizontal line array are proposed. The behavior of the angles, which are obtained for two episodes from the Shallow Water 2006 (SW06) experiment, show agreement with the theory presented.

(c) 2018 Acoustical Society of America. https://doi.org/10.1121/1.5020274

[JAC]

Pages: 193-201

\section{INTRODUCTION}

A variability of the oceanic waveguide's parameters in the horizontal plane (due to bathymetry or the sound speed profile) lead to the set of effects in sound propagation, which are called "horizontal refraction," or more generally "3D effects." These effects have been studied theoretically and experimentally both in the deep ocean (Collins et al., 1995) and in shallow water (Heaney and Murray, 2009; Katsnel'son et al., 2007). As has been shown in numerous past papers, there are a number of acoustical manifestations of horizontal refraction due to coastal wedges. The first observation and measurement of horizontal angle effects was carried out in a coastal wedge by (Doolittle et al., 1988). Subsequently, the following phenomena were studied: focusing/defocusing of the sound field in the horizontal plane in the presence of nonlinear internal waves (Katsnelson and Pereselkov, 2000; Badiey et al., 2005); variations of the interference pattern in the horizontal plane in the area of a coastal wedge in (Deane and Buckingham, 1993; Katsnelson et al., 2013), or in the area of the underwater canyon in (Duda et al., 2011; Y.-T. Lin, 2013); multipath propagation in the horizontal plane in an experiment in the Florida Strait (Heaney and Murray, 2009); and many others. In the papers (Bender et al., 2014; Ballard et al., 2012), the importance of taking horizontal refraction into account for inverse problems was discussed as well.

The quantitative description of sound propagation in shallow water in the presence of horizontal inhomogeneities can be based upon the decomposition of the complex

a)Electronic mail: bkatsnels@univ.haifa.ac.il amplitude of the sound field $P(\mathbf{r}, z)$ at some frequency $\omega$ into waveguide modes (vertical modes) $\psi_{n}(z ; \mathbf{r})$ which depend upon the horizontal coordinates $\mathbf{r}=(x, y)$, as well as on vertical parameters $(z$ is the vertical coordinate directed downward, and $z=0$ corresponds to the surface). Modal amplitudes $A_{n}(\mathbf{r}, \omega)$ in the sound field decomposition satisfy a wave equation which can be solved either in the ray approximation (horizontal rays and vertical modes) (Weinberg and Burridge, 1974) or using the parabolic equation method (Collins et al., 1995; Colosi, 2016).

One of the key features of these phenomena is the frequency dependence of the modal amplitude $A_{n}(\mathbf{r}, \omega)$ (in particular, the amplitude and phase of the horizontal rays) and, in turn, the interference pattern of the sound field in the horizontal plane. In other words, after separating out the vertical coordinate $z$, a two-dimensional ( $x-y$ plane) wave equation is obtained, which describes wave propagation in a twodimensional dispersive medium.

Given this background, the goal of this paper is to study the specific physical features of the interference pattern due to its frequency dependence or, more specifically, the behavior of the phase and amplitude fronts of a sound pulse propagating in a shallow water waveguide. Such frequency dependence in the presence of horizontal refraction plays significant role in shallow water acoustics (Katsnelson and Pereselkov, 2004; Dong et al., 2015).

\section{PHASE AND AMPLITUDE FRONTS IN THE INTERFERENCE PATTERN}

Let us consider a narrow-band sound signal propagating from a point source in shallow water with parameters (for example, the sound speed profile and/or bathymetry) 
depending on the horizontal $(x-y)$ coordinates, and being received by some receiving system. The complex sound field, which depends on both time and the spatial point $(\mathbf{r}, z)$ has the form

$$
P(\mathbf{r}, z, t)=\int \sum_{n} A_{n}(\mathbf{r}, \omega) \psi_{n}(z ; \mathbf{r}, \omega) \exp (-i \omega t) d \omega,
$$

where $\psi_{n}(z ; \mathbf{r}, \omega)$ are the waveguide modes, which depend weakly on the horizontal coordinates as adiabatic modes and smoothly upon the frequency (excluding the region near the cutoff frequency). The corresponding modal eigenvalues $q_{n}$ (propagation constants) depend on $\mathbf{r}$ and $\omega$ as well: $q_{n}=q_{n}(\mathbf{r}, \omega)$.

If the spectrum of the signal is narrow enough, i.e., $\omega_{1}<\omega<\omega_{2}$, where $\Delta \omega=\omega_{2}-\omega_{1} \ll \omega$, then the eigenfunction, due to the smooth frequency dependence of the mode shape, can be taken outside the integral (1) at some central frequency $\omega_{1}<\omega_{0}<\omega_{2}$,

$$
\begin{aligned}
P(\mathbf{r}, z, t) & =\sum_{n} \psi_{n}\left(z ; \mathbf{r}, \omega_{0}\right) \int A_{n}(\mathbf{r}, \omega) \exp (-i \omega t) d \omega \\
& =\sum_{n} \psi_{n}\left(z ; \mathbf{r}, \omega_{0}\right) P_{n}(\mathbf{r}, t),
\end{aligned}
$$

where we have introduced the modal amplitude, which depends on the horizontal coordinates and time, as

$$
P_{n}(\mathbf{r}, t)=\int A_{n}(\mathbf{r}, \omega) \exp (-i \omega t) d \omega .
$$

Neglecting mode coupling, $A_{n}(\mathbf{r}, \omega)$ satisfies the twodimensional Helmholtz equation where the wave number is $q_{n}(\mathbf{r}, \omega)$. Using standard techniques, it is possible to find the spectral amplitude $A_{n}(\mathbf{r}, \omega)$ in the ray approximation

$$
A_{n}(\mathbf{r}, \omega)=a_{n}(\mathbf{r}, \omega) \exp \left[i s_{n}(\mathbf{r}, \omega)\right],
$$

where $s_{n}(\mathbf{r}, \omega)$ is the eikonal in the horizontal plane, corresponding to the mode $n$, which can be found from the two dimensional eikonal equation

$$
\left[\nabla_{\perp} s_{n}(\mathbf{r}, \omega)\right]^{2}=q_{n}^{2}(\mathbf{r}, \omega)
$$

where $\nabla_{\perp}=(\partial / \partial x, \partial / \partial y)$ is the gradient in the horizontal plane.

Due to the frequency dependence of the eigenvalues on the right side of Eq. (5), the horizontal rays have different paths for different frequencies On the basis of ray theory, it is possible to find horizontal rays joining the source and receiver (the so-called eigenrays). The frequency dependence of the ray path is the main peculiarity of the ray pattern in our given case, in other words eigenrays corresponding to different frequencies have different trajectories [Fig. 1(a)].

Next, we will analyze the sound field of a particular mode with index number $n$; for brevity, we will omit showing this index number explicitly in the following discussion. If we introduce a vector $\mathbf{q}:(|\mathbf{q}|=q)$, tangent to the horizontal ray path, then at the locations of the source and receiver, the vectors $\mathbf{q}(\omega)$, corresponding to different frequencies, will be different both in modulus and direction. In the Fig. 1(a), the vectors $\mathbf{q}_{1}$ and $\mathbf{q}_{2}$ are shown for frequencies $\omega_{1}$ and $\omega_{2}$ at the location of the receiver.

It is seen that, for the sound field of a narrowband signal in the neighborhood of the receiver, an interference pattern is formed. For this pattern, a rather simple illustration can be presented for the superposition of two horizontal rays (locally plane waves). To illustrate the interference pattern in the horizontal plane formed by two such rays with wave vectors $\mathbf{q}_{1}$ and $\mathbf{q}_{2}$, corresponding to two close frequencies $\omega_{1}$ and $\omega_{2}$ [Fig. 1(a)], also with equal amplitudes for simplicity, coming into the receiver, we write the sound field as

$$
\begin{aligned}
P(\mathbf{r}, t)= & A \exp \left[i\left(\mathbf{q}_{1} \mathbf{r}-\omega_{1} t+\varphi_{1}\right)\right] \\
& +A \exp \left[i\left(\mathbf{q}_{2} \mathbf{r}-\omega_{2} t+\varphi_{2}\right)\right] \\
= & A(\mathbf{p r}-t) \exp [i(\mathbf{q} \mathbf{r}-\omega t+\varphi)],
\end{aligned}
$$

where $\varphi_{1}$ and $\varphi_{2}$ are some phases, $\mathbf{q}=\left(\mathbf{q}_{2}+\mathbf{q}_{1}\right) / 2$, $\omega=\left(\omega_{2}+\omega_{1}\right) / 2, \mathbf{p}=\Delta \mathbf{q} / \Delta \omega, \varphi=\left(\varphi_{2}+\varphi_{1}\right) / 2, \Delta \mathbf{q}=\mathbf{q}_{2}$ $-\mathbf{q}_{1}, \Delta \omega=\omega_{2}-\omega_{1}, \Delta \varphi=\varphi_{2}-\varphi_{1}$, and $A(\mathbf{p r}-t)=2 A$ $\cos [(\Delta \omega(\mathbf{p r}-t)+\Delta \varphi) / 2]$.

So, in the vicinity of the receiver the sound field is modulated in space and time, with carrier frequency $\omega$ and complex amplitude $A(\mathbf{p r}-t)$. The scales of this modulation in the horizontal plane, i.e., the interference pattern similar to Fig. 1(b), depends upon the parameters of the waveguide. Let us now introduce a phase front (lines of constant phase) $\mathbf{q} \mathbf{r}-\omega t=$ constant, an amplitude front (line of constant amplitude) $\mathbf{p r}-t=$ constant, and normals to these lines, i.e., the vectors $\mathbf{q}$ (the direction of propagation of the phase front) and $\mathbf{p}$ (the direction of propagation of the amplitude front).

If the spectrum of the source is contained in the band $\omega_{1}<\omega^{\prime}<\omega_{2}$, then the wave vectors of the horizontal rays coming into the receiver will be located in the sector between

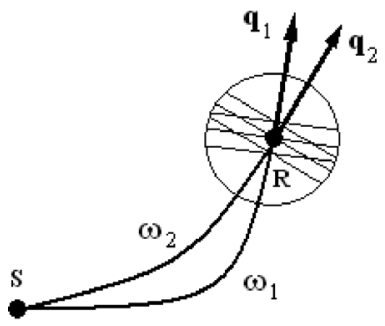

(a)

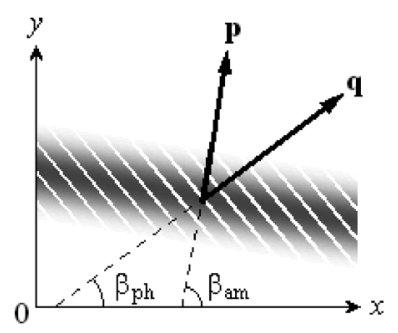

(b)

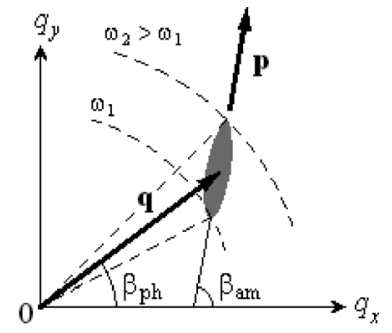

(c)
FIG. 1. (a) Two horizontal rays for different frequencies coming into a receiver. (b) Directions of the amplitude and phase fronts (wave vectors $\mathbf{p}$ and $\mathbf{q}$ ). (c) Spatial Fourier transform in the horizontal plane. 
$\mathbf{q}_{1}$ and $\mathbf{q}_{2}$. We can get an expression for the received signal by integration of Eq. (3) assuming a narrow spectrum where we can use a linear decomposition of the wave vector: $\mathbf{q}^{\prime}$ $=\mathbf{q}\left(\omega^{\prime}\right)=\mathbf{q}(\omega)+\mathbf{p}(\omega)\left(\omega^{\prime}-\omega\right)=\mathbf{q}+\mathbf{p}\left(\omega^{\prime}-\omega\right)$, where $\omega$ is the carrier frequency, for example $\omega=\left(\omega_{2}+\omega_{1}\right) / 2$, $\mathbf{p}(\omega)=d \mathbf{q} / d \omega$. So the received signals have the form

$$
\begin{aligned}
P(\mathbf{r}, t) & =\int_{\omega_{1}}^{\omega_{2}} A\left(\mathbf{r}, \omega^{\prime}\right) \exp \left[i\left(\mathbf{q}^{\prime} r-\omega^{\prime} t+\varphi^{\prime}\right)\right] d \omega^{\prime} \\
& =A(\mathbf{p r}-t) \exp [i(\mathbf{q r}-\omega t)],
\end{aligned}
$$

where the amplitude is $A(\mathbf{p r}-t)=\int_{\omega_{1}}^{\omega_{2}} A\left(\mathbf{r}, \omega^{\prime}\right) \exp$ $\left[i\left(\omega^{\prime}-\omega\right)(\mathbf{p r}-t)+i \varphi^{\prime}\right] d \omega^{\prime}$. In particular, assuming that within this band the spectral amplitude is constant, i.e., $A\left(\mathbf{r}, \omega^{\prime}\right)=A$, we get

$$
A(\mathbf{p r}-t)=2 A \Delta \omega \frac{\sin [\Delta \omega(\mathbf{p r}-t)]}{\Delta \omega(\mathbf{p r}-t)} .
$$

The time duration of the envelope of this signal is $\sim 2 \pi / \Delta \omega$, and the spatial scale of the envelope in the direction of propagation is $\sim 2 \pi /|\Delta \mathbf{q}|$. The interference pattern of the signal in the horizontal plane has rather complex character when the lines of constant amplitude have a different direction than the lines of constant phase. Waves of this type are called inhomogeneous (Born and Wolf, 1968), and are similar to those presented in Fig. 1(b). [A similar interference pattern has been observed for a wedge model by Katsnelson et al. (2013).]

We note that if the spectral components in Eqs. (6) and (7) have different amplitudes, then the result of their summation has a more complex form than in the right side of Eqs. (7) or (8), and in that case we will define the amplitude front as a line (or some area in the horizontal plane) of maximal amplitude.

\section{THEORETICAL ESTIMATES OF AMPLITUDE AND PHASE FRONT VARIATIONS}

Let us consider theoretical and numerical estimations of the possible angles for the amplitude front (AF) and phase front $(\mathrm{PF})$ directions within the framework of a simplified model for typical shallow water conditions.

As an example, consider sound propagation in the Shallow Water 2006 experiment (Newhall et al., 2007) in the presence of linear internal waves with small amplitude, having some anisotropy, and propagating mainly toward the coast. This situation has been seen to take place before and after the passage of a train of intense nonlinear internal waves [see, for example, Sabinin and Serebryani (2005)].

Let the source and receiver be placed at a distance $D$ relative to each other along the $r$ axis (Fig. 2), which is approximately parallel to the coastal line. For SW06, $D \approx 2 \times 10^{4}$ $\mathrm{m}$. The unperturbed sound speed profile $c(z)$ is characterized by a rather sharp thermocline, with thickness much less than the water depth, that is $h_{t} \ll H$ (for SW06 $h_{t} / H \sim 0.1$ ). The displacement of the isodensity surface from its equilibrium shape (plane) $\zeta(\mathbf{r}, z)$ characterizes the internal waves and the corresponding variation of the sound speed profile $c(\mathbf{r}, z)$

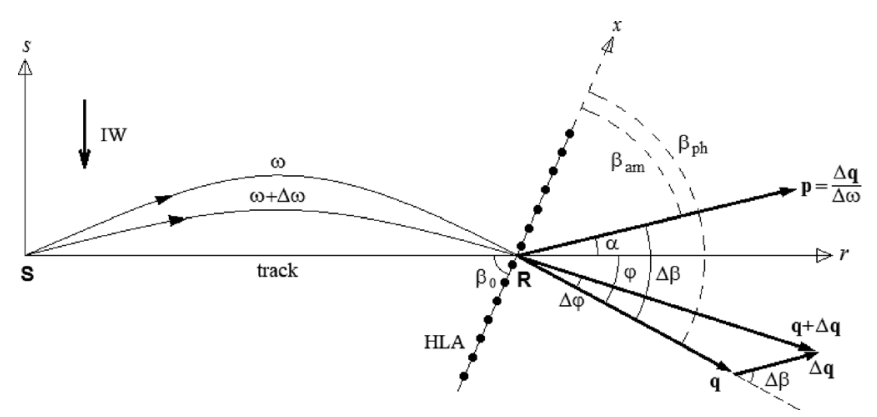

FIG. 2. Eigenrays joining source $\mathrm{S}$ and receiver $\mathrm{R}$ for two frequencies. We define positive angles in the counterclockwise direction. IW denotes the direction of motion of an internal wave. Estimates based upon references (Katsnelson et al., 2012; Katsnelson et al., 2013; Weinberg and Burridge, 1974) give: $|\varphi| \sim|\alpha| \sim 1-2^{\circ},|\Delta \beta| \sim 1-4^{\circ},|\Delta \varphi| \sim 0.1^{\circ}$ for $\Delta \omega \sim 10 \mathrm{~Hz}$.

$=c(z)+\delta c(\mathbf{r}, z)$. The correction to the sound speed profile can be written (Flatte, 1979)

$$
\frac{\delta c(\mathbf{r}, z)}{c(z)}=Q N^{2}(z) \zeta(\mathbf{r}, z)
$$

where $N(z)$ is the Brunt-Vaisala frequency (buoyancy frequency), and the parameter $Q$ depends on the water column properties. In shallow water $Q \sim 2.4 \mathrm{~s}^{2} / \mathrm{m}$.

For linear internal waves, the gradient of the displacement in the horizontal plane $\gamma=\nabla_{\perp} \zeta(\mathbf{r}, z)$ has the value $|\gamma| \sim 0.01$ and the perturbation of the sound speed in the area of the thermocline due to the displacement is $|\delta c| \sim 1 \mathrm{~m} / \mathrm{s}$.

The eigenvalue of the $n$th acoustic mode $q(\mathbf{r}, \omega)$ in the presence of the internal waves can be found using perturbation theory

$$
q(\mathbf{r}, \omega)=q^{0}(\omega)+\delta q(\mathbf{r}, \omega)
$$

where the first order correction $\delta q(\mathbf{r}, \omega)$ has the form (Katsnelson et al., 2012)

$$
\delta q(\mathbf{r}, \omega)=-\frac{Q k^{2}}{q^{0}} \zeta_{0}(\mathbf{r}) \int_{0}^{H}\left[\psi^{0}(z)\right]^{2} N^{2}(z) \Phi(z) d z,
$$

where $\zeta(\mathbf{r}, z)=\zeta_{0}(\mathbf{r}) \Phi(z), \zeta_{0}(\mathbf{r})$ is the displacement of the point corresponding to maximal amplitude, $\Phi(z)$ is the first mode of the internal gravity wave normalized to its maximal value $\max \Phi(z)=1, \psi^{0}(z)$ and $q^{0}$ are the eigenfunction and the eigenvalue of the mode $n$ in the absence of an internal wave (mode number omitted), and $k=\omega / \tilde{c}$, where $\tilde{c}$ is some average representative value of the sound speed in water.

$\delta q(\mathbf{r}, \omega) / q^{0}(\omega)$ is the first order correction to the refractive index in the horizontal plane, determining the trajectory of the ray paths from Eq. (5), and depending on mode number and frequency.

Let us next suppose that the displacement $\zeta$ depends on a coordinate $s$, transverse to the acoustic track (Fig. 2). Further, in the area of the ray paths (which have small horizontal angles in our problem) this displacement depends only linearly on $s: \zeta(\mathbf{r}, z)=\gamma s$, in the sense of a Taylor expansion. In this case, the correction to the refraction index in horizontal plane has the form 


$$
\begin{aligned}
& \frac{\delta q(\mathbf{r}, \omega)}{q^{0}(\omega)} \approx-\varepsilon(\omega) s, \\
& \varepsilon=\gamma \frac{Q k^{2}}{\left(q^{0}\right)^{2}} \int_{0}^{H}\left[\psi^{0}(z)\right]^{2} N^{2}(z) \Phi(z) d z .
\end{aligned}
$$

In some cases, in particular for SW06, it is possible to approximate that the function under integral (13) is concentrated within the thermocline of thickness $h_{t}$. Rough estimation of the integral in Eq. (13) gives $N_{0}^{2} h_{t} / H$, where $N_{0}$ is the value of the Brunt-Vaisala frequency inside the thermocline layer (its maximal value). The Brunt-Vaisala frequency can be expressed via the vertical gradient of temperature $T: N^{2}(z)$ $=(g / \rho)(d \rho / d z)=-\beta_{T} g(d T / d z)$, where $g \approx 10 \mathrm{~m} / \mathrm{s}^{2}, \rho$ is the density of water, and $\beta_{T} \sim 1.3 \times 10^{-4}$. $1 / \mathrm{K}$ is the water thermal compressibility coefficient. The variation of salinity in our situation is negligible.

So, in the area of the thermocline, where the temperature gradient can be $\sim 1 \mathrm{~K} / \mathrm{m}$, the maximal value of the squared Brunt-Vaisala frequency is estimated as: $N_{0}^{2} \sim(1-1.5)$ $\times 10^{-3} \mathrm{~Hz}^{-2}$. Neglecting the frequency dependence, which is small for a narrow-band signal, $|\varepsilon| \sim|\gamma| Q N_{0}^{2} h_{t} / H \sim(2.5-4)$ $\times 10^{-6} \mathrm{M}^{-1}$.

All the angles we deal with are defined in Fig. 2. Positive values of angles are in the counterclockwise direction. It is possible to estimate the horizontal angle $\varphi$ of an arriving ray relative to the acoustic track (Fig. 2) using a well known expression for the ray cycle distance $D$ (Katsnelson et al., 2012), assuming a linear dependence of the refractive index on the coordinate $s$ only Eq. (12),

$$
D=-\frac{2 \tan \varphi}{\varepsilon} \approx-\frac{2 \varphi}{\varepsilon} .
$$

Thus, as a result

$$
\varphi \approx-\frac{D \varepsilon}{2} .
$$

For the distance $20 \mathrm{~km},|\varphi| \sim 1^{\circ}-2^{\circ}$. The sign of this angle is determined by the gradient of the soundspeed along the $s$ axis. So, in Fig. 2 we have $\varphi<0$ for $\partial v / \partial s>0$ (if $\varepsilon>0$ ).

Let us next find the angle between horizontal eigenrays corresponding to different frequencies for the same vertical mode. Vectors $\mathbf{q}(\omega)$ and $\mathbf{q}(\omega+\Delta \omega)$ come to the location of the receiver at different angles $\varphi(\omega)$ and $\varphi(\omega+\Delta \omega)$ $=\varphi(\omega)+\Delta \varphi$. The angle $\Delta \varphi$ can be estimated as

$$
\Delta \varphi=-\frac{D}{2} \frac{d \varepsilon}{d \omega} \Delta \omega .
$$

To estimate the value $d \varepsilon / d \omega$, we use the expression (Katsnelson et al., 2012) for the connection between the phase and group velocities of a waveguide mode, which in simplified form is

$$
\frac{q}{\omega} \frac{\partial q}{\partial \omega} \approx \frac{1}{\tilde{c}^{2}} .
$$

For the Pekeris model, Eq. (17) is an exact expression where in the right hand side one sees the sound speed in the water layer. In our more general case, the right side is an average value of the sound speed over the depth and Eq. (17) is an estimate. Next, using Eqs. (12) and (17) we see

$$
\begin{aligned}
\frac{d \varepsilon}{d \omega} & =-\frac{d}{d \omega}\left(\frac{1}{q} \frac{\partial q}{\partial s}\right)=\frac{1}{q^{2}} \frac{\partial q}{\partial \omega} \frac{\partial q}{\partial s}-\frac{1}{q} \frac{\partial}{\partial s}\left(\frac{\partial q}{\partial \omega}\right) \\
& \approx-\frac{2 \omega \varepsilon}{q^{2} \tilde{c}^{2}} \approx-\frac{2 \varepsilon}{\omega} .
\end{aligned}
$$

Thus we get for $\Delta \varphi$, from Eq. (16) corresponding to the frequency interval $\Delta \omega$,

$$
\Delta \varphi=\frac{D \varepsilon}{\omega} \Delta \omega=-\frac{2 \varphi}{\omega} \Delta \omega .
$$

It follows from Eq. (19) that for horizontal eigenrays which are close to straight lines, the arriving and outgoing angles decrease with increasing frequency.

For a distance of $20 \mathrm{~km}$, a main pulse frequency $f=\omega /$ $(2 \pi)=300 \mathrm{~Hz}$, and a frequency band $\Delta f=\Delta \omega /(2 \pi)=10 \mathrm{~Hz}$, we estimate that $|\Delta \varphi| \sim 0.1^{\circ}$, and we also can write $\Delta \varphi / \Delta f$ $\sim-0.01 \mathrm{deg} / \mathrm{Hz}$.

Next, let us estimate the angle $\Delta \beta$ between the amplitude and phase fronts (Fig. 2), or equivalently between the vectors $\mathbf{p}=\Delta \mathbf{q} / \Delta \omega$ and $\mathbf{q}$, using the triangle formed by the vectors $\mathbf{q}$ and $\mathbf{q}+\Delta \mathbf{q}$ for frequencies $\omega$ and $\omega+\Delta \omega$. In this case, taking into account the smallness of $\phi$,

$$
\Delta \beta \approx \frac{q}{\Delta q} \Delta \varphi \approx-2 \varphi \frac{q}{\Delta q} \frac{\Delta \omega}{\omega} .
$$

The factor containing $(q / \Delta q)(\Delta \omega / \omega)$ is close to the ratio of the modal group velocity to the modal phase velocity. If we estimate the group velocity to phase velocity ratio roughly as 1 , then $\Delta \beta \approx-2 \varphi$, which leads to the estimate of the angle between the phase and amplitude fronts as $|\Delta \beta| \sim 1-4^{\circ}$.

The angle $\alpha$ (Fig. 2), determining the direction of the amplitude front (vector $\mathbf{p}$ ) relative to the direction of the acoustic track, is

$$
\alpha=\varphi+\Delta \beta,
$$

or roughly $\alpha \approx-\varphi$. It is interesting to note that the normal vector to the amplitude front is directed to the other side, relative to the acoustic track, than the normal vector to the phase front. We remark that this is the result of the rather idealized model used for our estimates; in a more realistic situation the ray pattern, and in turn the connection between angles, can be much more complex.

We should also note that the above-mentioned estimates, which display increasing effects of horizontal refraction with decreasing frequency, are obtained assuming a linear dependence of the displacement $\zeta$ and are valid for ray paths in this assumed region.

\section{A METHOD TO FIND THE ANGLE BETWEEN THE AMPLITUDE AND PHASE FRONTS FROM EXPERIMENTAL DATA, AND ANALYSIS OF SW06 RESULTS}

Let us now consider how to find the values of the aforementioned angles on the basis of experimental acoustical 
data. Information about the details of the interference pattern in the horizontal plane (for example, the directions of the normals to the amplitude front and the phase front) can be obtained using a spatial two-dimensional Fourier transform (FT). For fixed time and depth, for example near the bottom, the amplitude of the sound field is $P\left(\mathbf{r}, H, t_{0}\right)=P(x, y)$ (suppressing depth $H$ and time $t_{0}$ ) and the FT has the form

$$
G\left(q_{x}, q_{y}\right)=\left|\iint P(x, y) \exp \left[-i\left(q_{x} x+q_{y} y\right)\right] d x d y\right| .
$$

A typical spatial FT in the plane $\left(q_{x}, q_{y}\right)$ for the sound field formed by close horizontal rays is shown in Fig. 1(c), where the parameters of the problem (vectors $\mathbf{q}$ and $\mathbf{p}$ ) and the corresponding angles are denoted.

The spatial spectrum produced by Eq. (22) is a result of integrating the sound field in the horizontal plane, but by using dispersion relationships for the waveguide modes it is possible to get $G\left(q_{x}, q_{y}\right)$ directly from experimental data measured by an L-shaped array.

Let us denote direction of the horizontal line array (HLA) as the $x$ axis, which means that the set of observational data can be represented as the function $P(x, t)$. As the first step, we construct the space-time spectrum of the received signal depending on the component $q_{x}$ and the frequency $\omega$,

$$
G\left(q_{x}, \omega\right)=\left|\iint P(x, t) \exp \left[-i\left(q_{x} x-\omega t\right)\right] d x d t\right|
$$

Let us next use the dispersion relation giving the connection between the components of the vector $\mathbf{q}$,

$$
\sqrt{q_{x}^{2}+q_{y}^{2}}=q(\omega)
$$

where $q(\omega)$, as a function of frequency, can be obtained from the Sturm-Liouville eigenvalue problem for the vertical crosssection at the receiver position. From Eq. (24), we can express frequency as a function of the components $\left(q_{x}, q_{y}\right)$ and after substitution into Eq. (23), it is possible to get $G\left(q_{x}, q_{y}\right)$ and in turn to estimate all the aforementioned angles.

We next apply this methodology to data processing and analysis of the SW06 results.
As an example, consider two episodes from the Shallow Water 2006 experiment. The layout of the experiment is shown in Fig. 3. NRL300 is the Naval Research Laboratory source, fixed near the bottom. It radiated linear frequency modulated (LFM) signals: the duration of each one was $2.048 \mathrm{~s}$, the frequency band was $270-330 \mathrm{~Hz}$, and the time period of radiation was $4.096 \mathrm{~s}$. The receiver was an Lshaped array: the horizontal part of it (the horizontal line array, or HLA) was of length $L=465 \mathrm{~m}$ and contained 32 hydrophones spaced by $15 \mathrm{~m}$. Its direction is the $x$ axis, as seen in Fig. 3, with $-L / 2 \leq x \leq L / 2$. The vertical part of the array (the vertical line array, or VLA) contained 16 hydrophones, and its horizontal coordinates are given by $(L /$ $2,0)$. The angles in the horizontal plane determining the orientation of the HLA relative to the acoustic track are shown in Fig. 3(a): $\beta_{1}=25.75^{\circ}, \beta_{0}=26.04^{\circ}, \beta_{2}=26.34^{\circ}$. The depth of the sea along the acoustic track was $\approx 80 \mathrm{~m}$.

A strong characteristic of the experimental region is the existence of nonlinear internal waves (NIW) with amplitudes $5-25 \mathrm{~m}$, propagating approximately toward the coast (perpendicular to the acoustic track). These arise at the M2 tidal period (roughly two times per day) and form the dominant anisotropy of the internal wave (IW) field. In this paper, two transmission time intervals from 19 of August 2006 are considered: 10:01-10:08 Greenwich mean time (GMT) (Fig. 4) and 08:30-08:38 GMT. Variations of the sound speed profile are determined by three thermistor strings, denoted SW45, SW54, and SW32, deployed approximately along the acoustic track.

Let us first consider the time period 10:01-10:08 GMT. In Fig. 5(a), thermistor records for this period are shown, with the instruments placed in the lower part of the thermocline. As we can see, vertical oscillations of the thermocline layer in area of the source and receiver are seen with amplitude $\sim 1 \mathrm{~m}$; in the middle part of acoustic track, their amplitude is $\sim 3 \mathrm{~m}$. In Fig. 3(b), the amplitude of one of the pulses $|P(x, t)|$ received by the HLA is shown after matched filtering. ("Matched filtering" means that the spectrum of the received signals is multiplied by the complex conjugate spectrum, which leads to the compression of the received LFM pulse from $\sim 2$ to $0.03 \mathrm{~s}$.) As a result, the signal-tonoise-ratio (SNR) is increased by about 70 times, and this makes it possible to separate modes using arrival times. In Fig. 3(b) we can see two dark lines corresponding to modes 3 and 4. The shape of the intensity distribution on the VLA

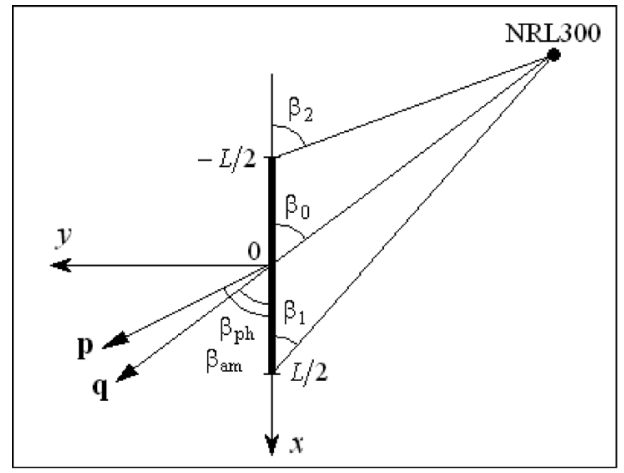

(a)

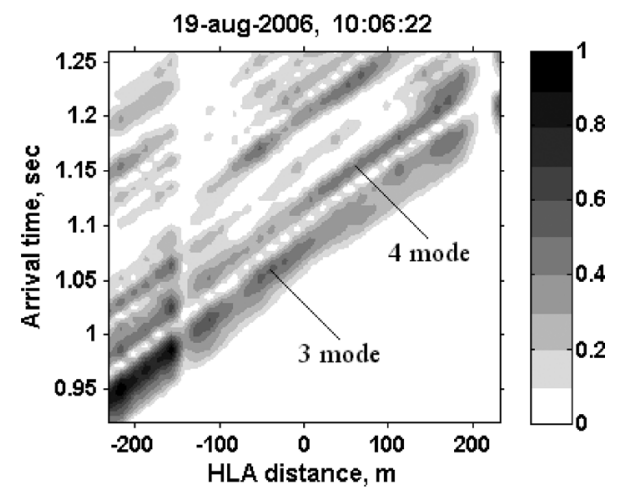

(b)
FIG. 3. (a) Scheme of the SW06 experiment. (b) Amplitude of one pulse, received by the HLA after matched field compression, showing arrival time dependence. 


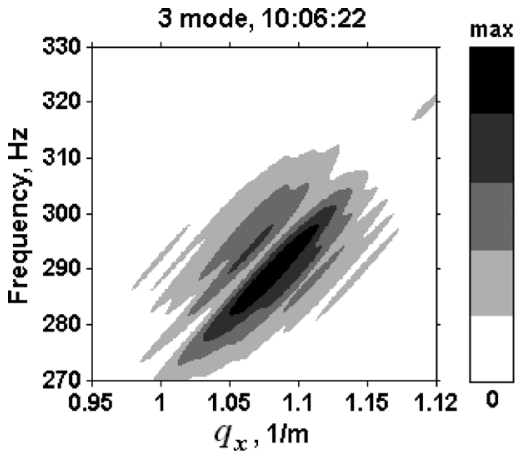

(a)

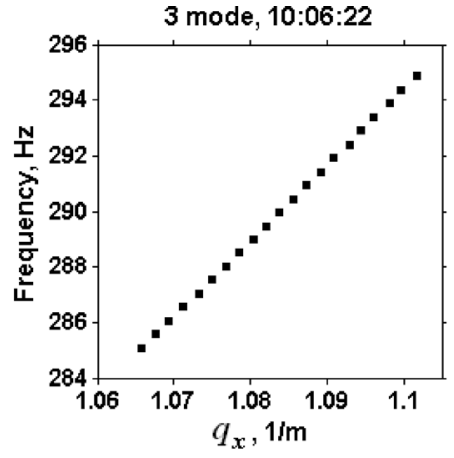

(b)

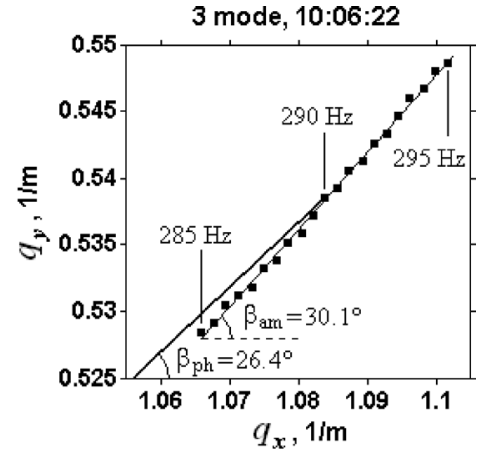

(c)

FIG. 4. Data processing. (a) Space-time spectrum of mode 3; the amplitude is shown in Fig. 3(b). (b) Filtering of spectral maxima for frequencies in the band $290 \pm 5 \mathrm{~Hz}$. (c) Calculation of the spatial spectrum in coordinates $\left(q_{x}, q_{y}\right)$ using the space-time spectrum.

can be used to determine the mode number: for mode 3, there are 3 maxima, and for mode 4 , there are 4 maxima.

We note that the maximal compression for the $60 \mathrm{~Hz}$ band, which is $1 / 60 \approx 0.02 \mathrm{~s}$, was 1.5 times less than that observed in the experiment, probably due to different phase shifts for different frequency components provided by intramodal dispersion, and due to different phase shifts along different horizontal rays corresponding to different frequencies.

(a)
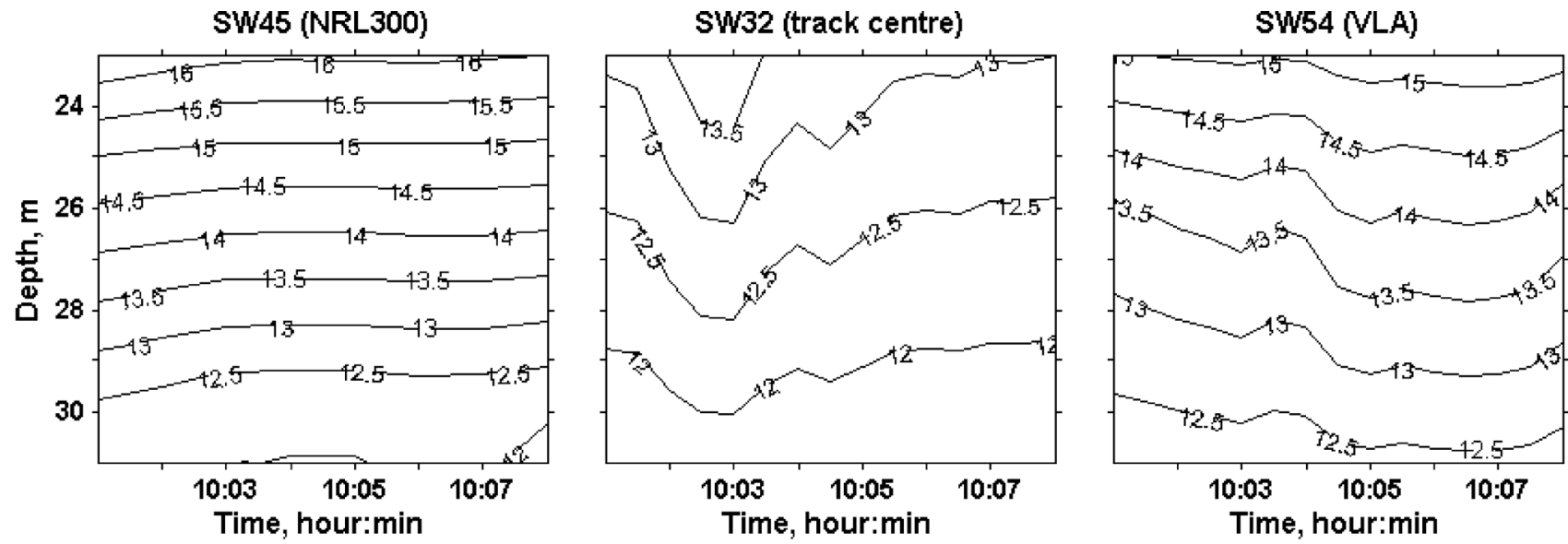

19 aug 2006,3 mode

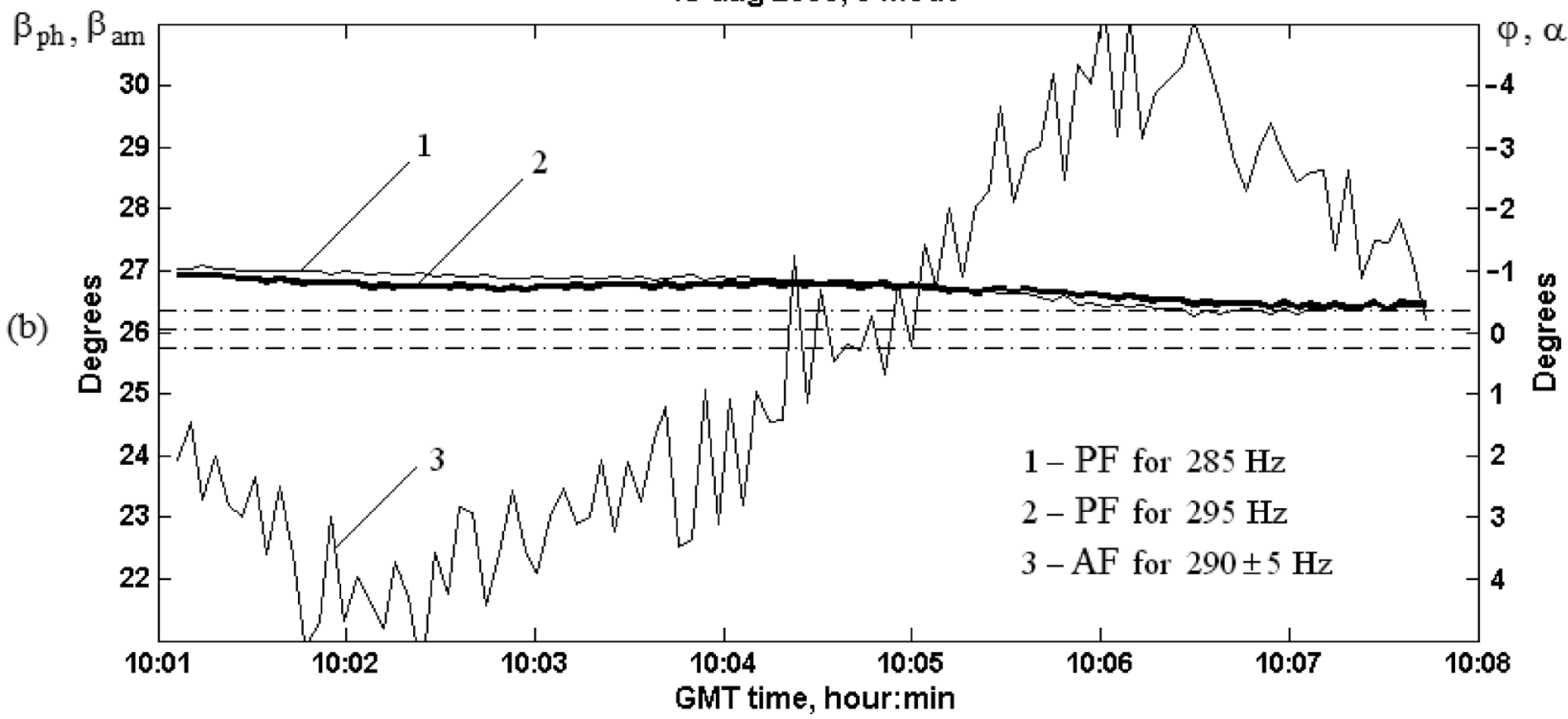

FIG. 5. Episode 10:01-10:08 GMT. (a) Thermistor records (in degrees Centigrade) on SW45, SW32, and SW54. These were located at: the NRL300, in the middle of acoustic track and at the receiver (VLA). (b) Temporal variations of the amplitude front and the phase front. The three dotted lines correspond to angles $\beta_{1}, \beta_{2}$, and $\beta_{0}$, giving the directions from the source to the ends and middle point of HLA [Fig. 3(a)]. Values of angles for amplitude and phase fronts are denoted by numbers for the frequencies shown in the figure. 
Let us consider the following analysis for mode 3. In Fig. 4(a), the space-time spectrum $G\left(q_{x}, \omega\right)$ is shown, calculated using Eq. (23) for mode 3 and for one of received pulses $P(x, t)$. The next step is to get the spatial spectrum, similar to Fig. 1(c), using Eq. (24), and to find the direction of the vector $\mathbf{p}$ in coordinates $\left(q_{x}, q_{y}\right)$ using a linear interpolation of the points corresponding to $\max \left|G\left(q_{x}, q_{y}\right)\right|$. In Fig. 4(b), the maxima of the spectrum $G\left(q_{x}, \omega\right)$ are shown, obtained for frequencies in the band $290 \pm 5 \mathrm{~Hz}$, which correspond to the neighborhood of the main maximum at $290 \mathrm{~Hz}$. Using the values of $\omega$ and $q_{x}$ for each maximum, we find $q_{y}$ from Eq. (24), where the dispersion law $q(\omega)$ and in turn $\omega(q)$ were obtained from the Sturm-Liouville problem using experimental data about the waveguide parameters at the location of the receiver. So, the positions of the maxima are found at the coordinates $\left(q_{x}, q_{y}\right)$, and also the straight line of maxima for the spatial spectrum $G\left(q_{x}, q_{y}\right)$ [Fig. 4(c)]. Angles $\beta_{\text {am }}$ and $\alpha=\beta_{0}-\beta_{\text {am }}$, characterizing the angle of the amplitude front with the HLA and the acoustic track (the angle between $\mathbf{p}$ and the axes $x$ and $r$ ), were found from the slope of the line of maxima relative to the axis $q_{x}$. Using the slope of the lines from the origin to the points corresponding to frequencies 285,290 , and $295 \mathrm{~Hz}$, we have found the angles $\beta_{\mathrm{ph}}$ and $\varphi=\beta_{0}-\beta_{\mathrm{ph}}$, which determine the angles between the phase front and the HLA and the acoustic track (angle between $\mathbf{q}$ and axes $x$ and $r$ ) for the given frequencies [Fig. 4(c)]. A frequency band of $10 \mathrm{~Hz}(290 \pm 5 \mathrm{~Hz})$ was chosen for calculations to insure the condition of linearity of the variation of the wave vector in this interval. Geometrically, this means that the vectors $\mathbf{q}=\left(q_{x}, q_{y}\right)$ in this frequency interval are placed in a straight line.

For the time interval 10:01-10:08 GMT, 98 pulses were processed. The results are shown in Fig. 5(b), where the temporal dependence of the angles characterizing the amplitude front and the phase front are presented. On the vertical axis on the left side, the values of $\beta_{\mathrm{ph}}$ and $\beta_{\mathrm{am}}$ are placed, and on the right side $\varphi$ and $\alpha$. These angles are shown for frequencies 285 and $295 \mathrm{~Hz}$.

Analysis of the angles obtained above leads to a good correspondence with the theoretical estimates (Fig. 2): the average value of the angle between the directions of the normal to the phase fronts for frequencies 285 and $295 \mathrm{~Hz}$ is seen to be $|\Delta \varphi| \approx 0.1^{\circ}$ in accordance with the estimate from Eq. (19); the average modulus of the angle between the directions of the amplitude front and the phase front for frequency $290 \mathrm{~Hz}$ is $|\Delta \beta|=\left|\beta_{\mathrm{ph}}-\beta_{\mathrm{am}}\right| \approx 2.9^{\circ}$ [Eq. (20)]; the average value of angle between the direction of the normal to the phase front and the acoustic track at frequency $290 \mathrm{~Hz}$ is $|\varphi| \approx 0.63^{\circ}$ [Eq. (15)]; the average value of the angle between the direction of the normal to the amplitude front and the acoustic track is $|\alpha| \approx 2.7^{\circ}$. We note that the fluctuations of the steering angle in SW06 that were reported in the paper by (Duda et al., 2012) for signals at $200 \mathrm{~Hz}$, using the coherence function of the horizontal line array in the presence of nonlinear internal waves, were of the same order.

Next, let us consider the following. At 10:05 GMT, the angle of the amplitude front $\alpha$ and two angles of the phase front $\varphi$ at the frequencies 285 and $295 \mathrm{~Hz}$ are equal to each other. In accordance with the simplified theory, this can only take place for straight-line trajectories of the horizontal eigenrays where $\beta_{\mathrm{am}}=\beta_{\mathrm{ph}}=\beta_{0}=26.04^{\circ}$. In the experiment, at 10:05 GMT we have $\beta_{\mathrm{am}} \approx \beta_{\mathrm{ph}} \approx 26.8^{\circ}$, that is greater than $\beta_{0}$ almost by $1^{\circ}$. This can be explained either by a systematic error in the measurement of angles, or by a more complex shape of the real IW than was supposed in the simplified theory. In the latter case, in spite of real horizontal refraction, the directions of the amplitude front and the phase front are the same in the neighborhood of the receiver. The systematic error can be produced by the deviations from a straight-line shape of HLA, as was discussed in (Duda et al., 2012).

Next, we note that, in accordance with the theory presented above, if direction of horizontal gradient of the sound speed profile is changed, then the signs of angles $\varphi$ and $\alpha$ are changed also. This leads to the following inequalities for angles $\beta_{\mathrm{ph} 1}$ and $\beta_{\mathrm{ph} 2}$, corresponding to 285 and $295 \mathrm{~Hz}$, and angle $\beta_{\mathrm{am}}: \beta_{\mathrm{am}}<\beta_{\mathrm{ph} 2}<\beta_{\mathrm{ph} 1}$ if the gradient is directed toward positive values of $s$, and $\beta_{\mathrm{am}}>\beta_{\mathrm{ph} 2}>\beta_{\mathrm{ph} 1}$, in the case of the opposite gradient direction.

In the experiment at time 10:05 GMT, a change of the sign of the angles takes place that can be interpreted as a change of the direction of gradient of the sound speed or a change of the thermocline slope (e.g., the forward face or back face of an internal wave).

Let us consider the variations of angles $\beta_{\mathrm{am}}$ and $\beta_{\mathrm{ph}}$ together with the thermistor records. It is seen from Fig. 5(a) that, on average in the time interval 10:01-10:04 GMT, the forward front of the moving IW is passing through the acoustic track (SW45 shows an approximately constant level of thermocline displacement, whereas SW32 and SW54 show an increase in displacement). In this case, we should have deflection of the eigenrays toward the area $s>0$, and so the inequality $\beta_{\mathrm{am}}<\beta_{\mathrm{ph} 2}<\beta_{\mathrm{ph} 1}$ takes place (Fig. 2). In the interval 10:04-10:08 GMT, the character of the IW motion is changed; specifically, the thermistor string SW32 shows mainly a decrease of the thermocline's displacement, whereas SW45 and SW54 show an approximately constant level of displacement. Here, it is probable that the back edge of the IW is passing through the acoustic track, and the values of the angles correspond to: $\beta_{\mathrm{am}}>\beta_{\mathrm{ph} 2}>\beta_{\mathrm{ph} 1}$ [Fig. 5(b)].

Similar work was done for another time interval, 08:30-08:38 GMT, where 110 pulses were processed. Thermistor records are shown in Fig. 6(a). The variations of the angles determining the directions of the amplitude front and the phase front are shown in the Fig. 6(b).

It is seen that the properties of the angle variability have the same character as the first example. In particular, at 08:37 GMT, the directions of the amplitude front and the phase front are the same. Average values of the angles in this period are $|\Delta \varphi| \approx 0.07^{\circ},|\Delta \beta| \approx 2.1^{\circ},|\varphi| \approx 0.67^{\circ}$, $|\alpha| \approx 2.7^{\circ}$, in accordance with theoretical estimations.

Next, let us examine the angles $\beta_{\mathrm{ph}}$ and $\beta_{\mathrm{am}}$ for the first and second periods for frequencies 290 and $305 \mathrm{~Hz}$, taking into account our confidence/error interval. In the first case: $\beta_{\mathrm{ph}}=26.7^{\circ} \pm 0.2^{\circ}, \quad \beta_{\mathrm{am}}=26^{\circ} \pm 3^{\circ} ;$ in the second case: $\beta_{\mathrm{ph}}=26.7^{\circ} \pm 0.1^{\circ}, \beta_{\mathrm{am}}=28.8^{\circ} \pm 1.5^{\circ}$. These confidence/ error intervals correspond to the mean square deviations. As we can see, the mean square fluctuations of the amplitude front's angle are bigger than those of the phase front by 

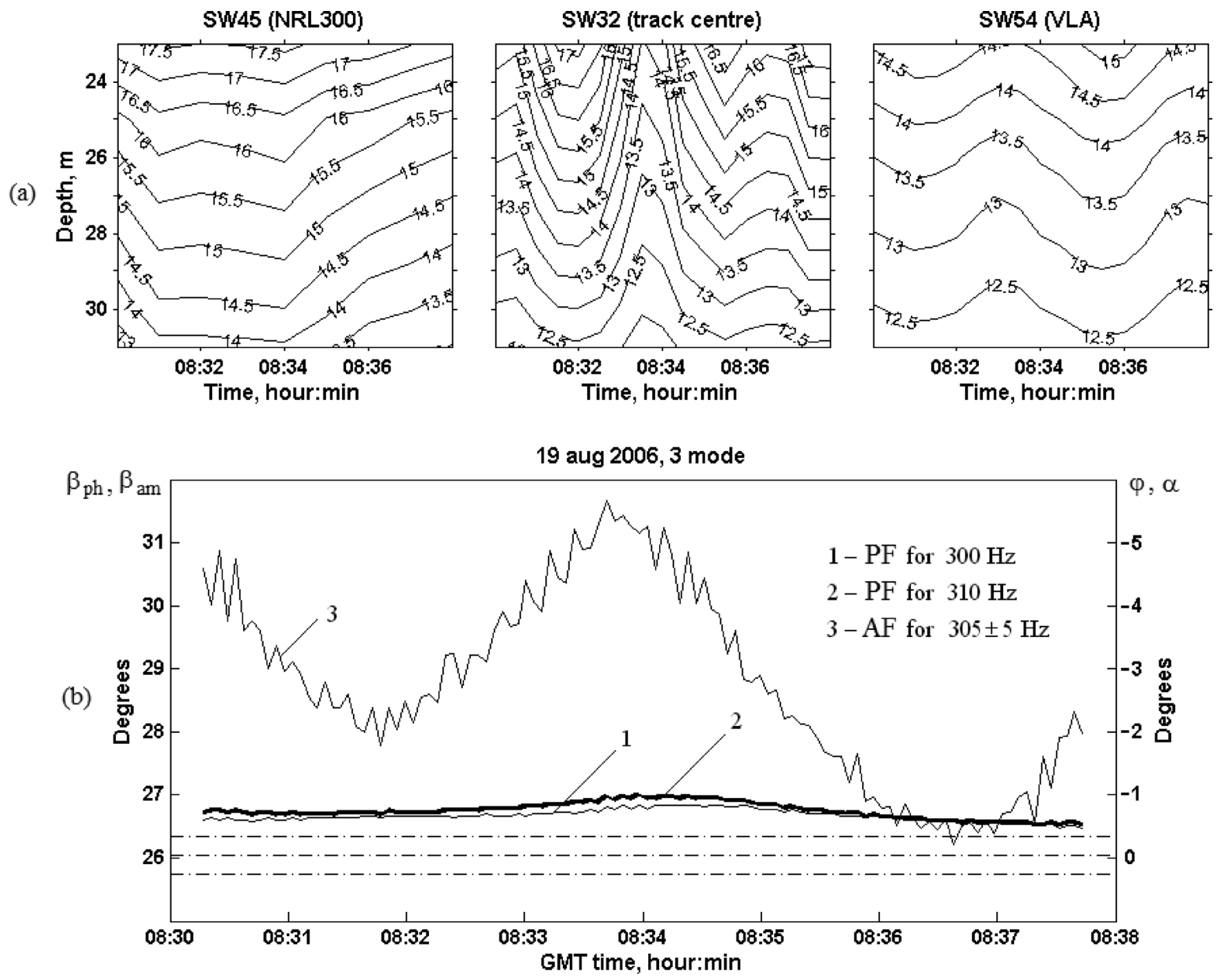

FIG. 6. Results of data processing for the period 08:30-08:38 GMT.

approximately by 15 times. In other words, the phase front is essentially more stable with respect to variations of the waveguide parameters in the horizontal plane than the amplitude front. This is a standard and expected result.

Finally, we should note that, in our theory, we neglect the coupling between vertical modes of different number which is determined by the ratio of matrix element $V_{m n} \sim \int \psi_{m}$ $\left(\partial \psi_{n} / \partial r\right) d z$ to the difference $\left|q_{m}-q_{n}\right|$. In our case this ratio $\kappa \sim V_{n m} /\left|q_{m}-q_{n}\right| \sim 10^{-3}$ for the closest pair of modes: 3-2 and 3-4. Thus, neglecting mode coupling is reasonable.

\section{CONCLUSIONS}

Let us present our conclusions as a simple list.

(1) It has been shown that the frequency dependence of trajectories of horizontal rays (or of the modal amplitude distribution in horizontal plane) leads to a specific interference pattern in the horizontal plane in the area of reception of the sound pulse. This pattern is interpreted as a combination of two structures: phase fronts (lines of constant phase) and amplitude fronts (lines of constant/ maximal amplitude or envelopes). These lines have different directions and different spatial scales of variability. We note that a similar interference pattern takes place in nonlinear and nano-optics for the propagation of two frequency beams in a crystal (Bakunov et al., 2012).

(2) On the basis of analytical estimations, it is shown that in a typical situation, if horizontal refraction is due to internal waves of small amplitude $(\sim 1 \mathrm{~m})$, then the angle of revolution of the phase front (direction of the horizontal ray) is about $\varphi \sim 1^{\circ}-2^{\circ}$ for an acoustical track of typical length $20 \mathrm{~km}$. The angle $\Delta \varphi$ between horizontal rays, corresponding to different frequencies, depends on the difference between the frequencies. Using the estimate $\Delta \varphi / \Delta \omega \sim-0.01 \mathrm{deg} / \mathrm{Hz}$, we get for a narrow-band pulse with bandwidth $10 \mathrm{~Hz}$ that this angle is approximately $\Delta \varphi \sim 0.1^{\circ}$ We note that our estimates should be modified in the presence of nonlinear internal waves (Duda et al., 2012) where experimental measurement of these angles is more difficult.

(3) The angle $\beta$ determining the direction of the normal to the amplitude front can be essentially greater than the angle determining the normal to the phase front. It implies a more remarkable manifestation of horizontal 
refraction than one would suppose using the trajectory of the horizontal rays. The variation of the direction of the amplitude front (or its sensitivity in the direction to waveguide parameter variations) is essentially higher than that for the phase front.

\section{ACKNOWLEDGMENT}

The authors are grateful to Professor O. Godin for helpful discussions. This work was supported by the Israel Science Foundation, Grant No. 565/15, and the Ministry of Education and Sciences of the Russian Federation, Grant No. 14.Z50.31.0037.

Badiey, M., Katsnelson, B. G., Lynch, J. F., Pereselkov, S., and Siegman, W. L. (2005). "Measurement and modeling of three-dimensional sound intensity variations due to shallow-water internal waves," J. Acoust. Soc. Am. 117(2), 613-625.

Bakunov, M. I., Tsarev, M. V., and Mashkovich, E. A. (2012). "Terahertz difference-frequency generation by tilted amplitude front excitation," Opt. Exp. 20(27), 28573-28585.

Ballard, M., Lin, Y.-T., and Lynch J. (2012). "Horizontal refraction of propagating sound due to seafloor scours over a range-dependent layered bottom on the New Jersey shelf," J. Acoust. Soc. Am. 131(4), 2587-2598.

Bender, C. M., Ballard, M. S., and Wilson, P. S. (2014). "The effects of environmental variability and spatial sampling on the three-dimensional inversion problem," J. Acoust. Soc. Am. 135(6), 3295-3304.

Born, M., and Wolf, E. (1968). Principles of Optics (Pergamon, Oxford).

Collins, M. D., McDonald, B. E., Heaney, K. D., and Kuperman W. A. (1995). "Three-dimensional effects in global acoustics," J. Acoust. Soc. Am. 97, 1567-1575.

Colosi, J. (2016). Sound Propagation through Stochastic Ocean (Cambridge University Press, London), 424 pp.

Deane, G. B., and Buckingham, M. J. (1993). "An analysis of the threedimensional sound field in a penetrable wedge with a stratified fluid or elastic basement," J. Acoust. Soc. Am. 93, 1319-1328.

Dong, H., Badiey, M., and Chapman, R. (2015). "Matched mode geoacoustic inversion of broadband signals in shallow water," J. Acoust. Soc. Am. 137, 2390.
Doolittle, R., Tolstoy, A., and Buckingham, M. J. (1988). "Experimental confirmation of horizontal refraction from a point source in a wedgeshaped ocean," J. Acoust. Soc. Am. 83(6), 2117-2125.

Duda, T., Collis, J., Lin, Y-T., Newhall, A., Lynch, J., and DeFerrari, H. (2012). "Horizontal coherence of low-frequency fixed-path sound in a continental shelf region with internal-wave activity," J. Acoust. Soc. Am. 131(2), 1782-1797.

Duda, T. F., Lin, Y.-T., Zhang, W. G., Cornuelle, B. D., Lermusiaux, P. F. J. (2011). "Computational studies of 3D Ocean sound fields in areas of complex seafloor topography and active ocean dynamics," edited by C.-F. Chen et al., in Proceedings of the 10th International Conference on Theoretical and Computational Acoustics, NTU, Taiwan, 12 pp.

Flatte, S. M., ed. (1979). Sound Transmission Through a Fluctuating Ocean (Cambridge University Press, London), 299 pp.

Heaney, K. D., and Murray, J. J. (2009). "Measurements of threedimensional propagation in a continental shelf environment," J. Acoust. Soc. Am. 125(3), 1394-1402.

Katsnelson, B., Malykhin, A., and Tckhoidze, A. (2013). "Propagation of wideband signals in shallow water in the presence of meso-scale horizontal stratification," Acoust. Australia 41(1), 45-83.

Katsnelson, B., and Pereselkov, S. (2000). "Low-frequency horizontal acoustic refraction caused by internal wave solitons in a shallow sea," Acoust. Phys. 46(6), 684-691.

Katsnelson, B., and Pereselkov, S. (2004). "Space-frequency dependence of the horizontal structure of a sound field in the presence of intense internal waves," Acoust. Phys. 50(2), 169-176 (2000).

Katsnelson, B., Petnikov, V., and Lynch, J. (2012). Fundamentals of Shallow Water Acoustics (Springer, New York), 540 pp.

Katsnel'son, B. G., Badiey, M., and Lynch, J. (2007). "Horizontal refraction of sound in a shallow water and its experimental observations," Acoust. Phys. 53(3), 313-325.

Lin Y.-T. (2013). "A higher-order tangent linear parabolic-equation solution of three-dimensional sound propagation," J. Acoust. Soc. Am. 134(2), EL251-EL257.

Newhall, A. E., Duda, T. F., Keith von der Heydt, Irish J. D., Kemp J. N., Lerner S. A., Liberatore S. P., Ying-Tsong Lin, Lynch J. F., Maffei A. R., Morozov, A. K., Shmelev, A. A., Sellers, C. J., and Witzell, W. E. (2007). "Acoustic and oceanographic observations and configuration information for the WHOI moorings from the SW06 experiment," Woods Hole Oceanog. Inst. Tech. Report.

Sabinin, K. D., and Serebryani, A. N. (2005). "Intense short-period internal waves in the ocean," J. Mar. Res. 63(1), 227-261.

Weinberg, H., and Burridge, R. (1974). "Horizontal ray theory for ocean acoustics," J. Acoust. Soc. Am. 55(1), 63-79. 